\title{
Charting the evolution of the ages and metallicities of massive galaxies since $z=0.7$
}

\author{
Anna Gallazzi ${ }^{1}$, Eric F. Bell ${ }^{2}$, Stefano Zibetti ${ }^{1}$, Daniel Kelson ${ }^{3}$ and \\ Jarle Brinchmann ${ }^{4}$ \\ ${ }^{1}$ Dark Cosmology Center, University of Copenhagen, Niels Bohr Institute, Juliane Maries Vej \\ 30, 2100 Copenhagen, Denmark \\ ${ }^{2}$ Department of Astronomy, University of Michigan, Ann Arbor, MI 48109, USA \\ ${ }^{3}$ Observatories of the Carnegie Institution of Washington, Pasadena, CA 91101, USA \\ ${ }^{4}$ Leiden Observatory, Leiden University, 2300RA, Leiden, the Netherlands
}

email: gallazzi@dark-cosmology.dk

\begin{abstract}
Detailed studies of the stellar populations of intermediate-redshift galaxies can shed light onto the processes responsible for the significant evolution of the massive galaxy population since $\mathrm{z}<1$. We have undertaken such a study by means of deep rest-frame optical spectroscopy with IMACS on Magellan on a sample of $\sim 80$ galaxies selected from CDFS to have stellar masses $>10^{10} M_{\odot}$ and redshift $0.65<\mathrm{z}<0.75$. We analyse stellar absorption line strengths and interpret them with a Monte Carlo library of star formation histories to derive constraints on mean stellar ages, metallicities and stellar masses. We present here the first characterization of the stellar mass-metallicity and stellar mass-age relations at $\mathrm{z} \sim 0.7$ and their evolution to the present-day.
\end{abstract}

Keywords. galaxies: evolution, galaxies: stellar content, galaxies: high-redshift

\section{Introduction}

In the local Universe bulge-dominated red-sequence galaxies contribute more than half of the total stellar mass and metal density (Bell et al. 2003, Baldry et al. 2004, Gallazzi et al. 2008). Half of the stellar mass density of this population has come into place in the past 8 billion years (Bell et al. 2004, Faber et al. 2007, Brown et al. 2007). Over the same epoch, the total star formation rate density, dominated by blue galaxies with masses between $10^{10}$ and $10^{11} M_{\odot}$, has declined by almost a factor of 10 (Hopkins \& Beacom 2006). Detailed studies of the stellar population properties of $\mathrm{z}<1$ galaxies (i.e. the progenitors), in combination with star formation diagnostics and morphological characterization, can shed light on the relative importance of various physical processes potentially responsible for the star formation quenching at $\mathrm{z}<1$ and the ensuing build-up of the red-sequence. So far only a few studies have addressed the evolution in stellar population properties in the epoch $0<\mathrm{z}<1$ and they are generally based on co-added spectra (e.g. Schiavon et al. 2006, Cool et al. 2008, Sánchez-Blázquez et al. 2009). Therefore, they cannot distinguish between the evolution of individual galaxies already on the red-sequence at intermediate redshift and the evolution of the population through addition of quenched blue galaxies.

\section{Sample and physical parameters estimates}

In order to tackle the issues above, we have obtained deep multi-object rest-frame optical spectroscopy with IMACS on Magellan for a representative sample of 77 massive galaxies at redshift $\mathrm{z} \sim 0.7$, allowing us to measure velocity dispersions and stellar absorption features and estimate the stellar population properties for individual objects. 
The sample has been selected from the CDFS field of the COMBO-17 survey (Wolf et al. 2004) to have redshift in the range $0.65<z<0.75$ and an apparent R-band magnitude brighter than 22.7. This magnitude cut allows us to map the red-sequence down to its completeness limit at $\mathrm{z}=0.7$ of $\mathrm{M}_{*} \sim 3 \times 10^{10} \mathrm{M}_{\odot}$ (Borch et al. 2006) and to sample the massive end of the blue-cloud. These galaxies have been observed with a single mask for a total exposure time of 10 hours, reaching an average $\mathrm{S} / \mathrm{N}$ of 15 per $\AA$ for the most massive galaxies and of 8 for lower mass ones. The spectra have a resolution of $6.25 \AA \mathrm{FWHM}$ $(\sim 3.4 \AA$ rest-frame) and span the wavelength range $6500-9500 \AA$, covering the most significant absorption features from the $4000 \AA$-break up to Fe5335. Moreover, this sample has multi-wavelength coverage from the UV to $24 \mu \mathrm{m}$, including HST imaging.

We measure velocity dispersions down to $\sim 100 \mathrm{~km} / \mathrm{s}$ for $70 \%$ of the sample, with an accuracy of $\lesssim 15 \%$ for $45 \%$ of the sample, using the pPXF code by Cappellari \& Emsellem (2004). Stellar absorption features are measured off the spectra corrected for nebular emission lines using PLATEFIT (Tremonti et al. 2004). We derive constraints on stellar mass, light-weighted age and stellar metallicity following the Bayesian approach developed by Gallazzi et al. (2005) to analyse SDSS spectra of local galaxies. We interpret an optimal set of absorption indices (D4000 $, \mathrm{H} \delta_{\mathrm{A}}+\mathrm{H} \gamma_{\mathrm{A}}, \mathrm{H} \beta,\left[\mathrm{Mg}_{2} \mathrm{Fe}\right]$, [MgFe]', the same used at low redshift and affected the least by $\alpha /$ Fe variations) with a large spectral library based on Bruzual \& Charlot (2003) models and Monte Carlo star formation histories and metallicities, and derive probability density functions of galaxy physical parameters. Stellar ages and masses are constrained with a typical accuracy of $0.1 \mathrm{dex}$, while stellar metallicity is constrained on average within 0.25 dex, with a stronger dependence on spectral $\mathrm{S} / \mathrm{N}$.

\section{Mass-metallicity and mass-age relations at $z=0.7$}

Locally, galaxies show clear average trends of increasing stellar age and metallicity with increasing mass. The scatter in these relations is lowest above the 'transition mass' of $3 \times 10^{10} M_{\odot}$, where red-sequence galaxies dominate (Gallazzi et al. 2005, Kauffmann et al. 2003). We observe a similar trend in the mean luminosity-weighted age of $z=0.7$ galaxies increasing with stellar mass from $\sim 2 \mathrm{Gyr}$ at $3 \times 10^{10} M_{\odot}$ to $\sim 5 \mathrm{Gyr}$ at $3 \times 10^{11} M_{\odot}$. The distribution in age is narrow at masses $>10^{11} M_{\odot}$, while the scatter increases by 0.1 dex below this mass, qualitatively consistent with an evolution with redshift of the characteristic transition mass (c.f. Pannella et al. 2009). This is shown in the upper panel of Fig. 1 where the $z=0.7$ galaxies are compared to the local relation from Gallazzi et al. (2005). As expected the overall distribution in stellar age of $z=0.7$ galaxies is shifted to younger ages than present-day galaxies. However, under the assumption of passive evolution, $z=0.7$ galaxies could only contribute to the oldest fraction of presentday massive galaxies. This means that passive evolution is not viable already at masses $>10^{11} M_{\odot}$ or that lower-mass $z=0.7$ galaxies must evolve to contribute to the younger end of present-day massive galaxies.

In the lower panel of Fig.1 we show the distribution in stellar metallicity as a function of stellar mass at $z=0.7$, compared to the local relation. In addition to stellar age, the data suggest a mild evolution also in stellar metallicity since $z=0.7$. Somewhat surprisingly, this is particularly the case for the most massive galaxies. Their specific star formation rate, estimated from UV and IR luminosities as in Bell et al. (2005), is typically lower than $3 \times 10^{-11} \mathrm{yr}^{-1}$, hence we do not expect these massive galaxies to enrich their stellar populations significantly. On the contrary, around and below $10^{11} M_{\odot}$, galaxies have higher specific SFR ( $\left.\gtrsim 10^{-10} \mathrm{yr}^{-1}\right)$ and a large scatter in stellar metallicity, with a fraction of them having metallicities comparable to local galaxies. These intermediatemass $z=0.7$ star-forming galaxies could contribute to the more metal-rich and younger 


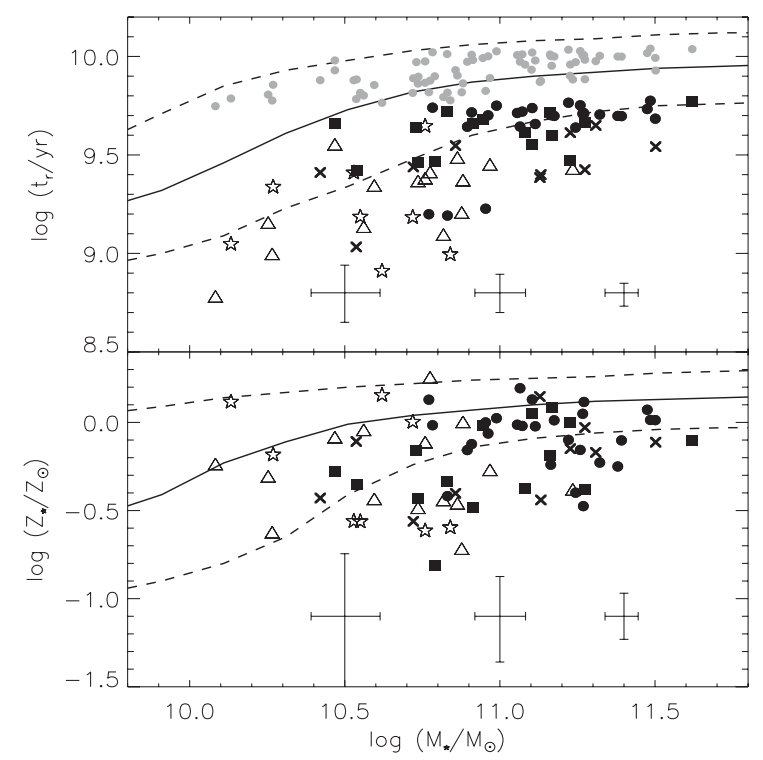

Figure 1. Luminosity-weighted age (upper panel) and stellar metallicity (lower panel) as a function of stellar mass for our sample galaxies at $z=0.7$. Average uncertainties are indicated for three mass bins. Different symbols reflect different values of specific star formation rates (circles: $\log \left(\mathrm{SFR} / \mathrm{M}_{*}\right)<-11$; squares: $-11<\log \left(\mathrm{SFR} / \mathrm{M}_{*}\right)<-10.5$; cross: $-10.5<\log \left(\mathrm{SFR} / \mathrm{M}_{*}\right)<-10$; traingles: $-10<\log \left(\mathrm{SFR} / \mathrm{M}_{*}\right)<-9.5$; stars: $\left.\log \left(\mathrm{SFR} / \mathrm{M}_{*}\right)>-9.5\right)$. Solid and dashed lines show the median and scatter of the local relations from Gallazzi et al. (2005). Grey points in the upper panel indicate the expected location of $z=0.7$ galaxies if they evolved passively until today.

fraction of present-day massive galaxies. Interestingly, this would be consistent with the age-metallicity anti-correlation observed at fixed mass, or velocity dispersion, in local quiescent galaxies (e.g. Graves et al. 2010, Gallazzi et al. 2006).

\section{References}

Baldry, I. K., Glazebrook, K., Brinkmann, J., et al. 2004, ApJ, 600, 681

Bell, E. F., McIntosh, D. H., Katz, N., et al. 2003, ApJS, 149, 289

Bell, E. F., Wolf, C., Meisenheimer, K., et al. 2004, ApJ, 608, 752

Bell, E. F., Papovich, C., Wolf, C., et al. 2005, ApJ, 625, 23

Borch, A., Meisenheimer, K., Bell, E. F., et al. 2006, A\& A, 453, 869

Brown, M. J. I., Dey, A., Jannuzi, B. T., et al. 2007, ApJ, 654, 858

Bruzual, G. \& Charlot, S. 2003, MNRAS, 344, 1000

Cappellari, M. \& Emsellem, E. 2004, PASP, 116, 138

Cool, R. J., Eisenstein, D. J., Fan, X., et al. 2008, ApJ, 682, 919

Faber, S. M., Willmer, C. N. A., Wolf, C., et al. 2007, ApJ, 665, 265

Gallazzi, A., Charlot, S., Brinchmann, J., et al. 2005, MNRAS, 362, 41

Gallazzi, A., Charlot, S., Brinchmann, J., et al. 2006, MNRAS, 370, 1106

Gallazzi, A., Brinchmann, J., Charlot, S., et al. 2008, MNRAS, 383, 1439

Graves, G. J., Faber, S. M., \& Schiavon, R. P. 2010, ApJ, 721, 278

Hopkins, A. M. \& Beacom, J. F. 2006, ApJ, 651, 142

Kauffmann, G., Heckman, T. M., White, S. D. M., et al. 2003, MNRAS, 341, 54

Pannella, M., Gabasch, A., Goranova, Y., et al. 2009, ApJ, 701, 787

Sánchez-Blázquez, P., Jablonka, P., Noll, S., et al. 2009, A\& A, 499, 47

Schiavon, R. P., Faber, S. M., Konidaris, N., et al. 2006, ApJ, 651, L93

Tremonti, C. A., Heckman, T. M., Kauffmann, G., et al. 2004, ApJ, 613, 898

Wolf, C., Meisenheimer, K., Kleinheinrich, M., et al. 2004, A\&্ A, 421, 913 